\title{
Range ambiguity clutter suppression for bistatic STAP radar
}

\author{
Wenchong Xie*, Baihua Zhang, Yongliang Wang, Yong Zhu, Keqing Duan and Rongfeng Li
}

\begin{abstract}
Bistatic pulse-Doppler airborne radar has desirable properties such as the low probability of detection by other radars relative to its monostatic counterpart. However, the clutter characteristics of bistatic airborne radar are more complex than those of monostatic airborne radar. The clutter spectra not only vary severely with range, but also vary with bistatic configuration. In this article, the geometry model of bistatic airborne radar is given, and the approximate estimation expressions for clutter degrees of freedom (DOFs) are presented. Then a novel clutter suppression method for bistatic airborne radar with range ambiguity is presented. The method completes registration-based range ambiguity clutter compensation based on non-uniform sampling and the estimated clutter DOFs. The simulation results illustrate the performance improvement achieved for bistatic airborne radar.
\end{abstract}

Keywords: Bistatic airborne radar, Non-stationarity, Range ambiguity, Space-Time Adaptive Processing (STAP), Clutter Degrees of Freedom (DOFs)

\section{Introduction}

Bistatic radar uses antennas at separate sites for transmission and reception, and it has been designed, developed, tested, and in some cases, deployed for military, commercial, and scientific applications. Typical military applications include air and space surveillance and range instrumentation. Commercial applications include wind field measurements and traffic surveillance. Scientific applications include measurement of planetary surfaces and atmospheres and study of ionospheric turbulence.

Advanced airborne-phased array radar must have the capability of suppressing wide and strong clutter effectively in order to detect the low-flying target in far field. In the last 30 years of research and development, space-time adaptive processing (STAP) [1-3] has become a new technique to be applied for airborne-phased array radar because the STAP processor can effectively suppress strong clutter and detect small target. Interest in bistatic STAP, where the

\footnotetext{
* Correspondence: xwch1978@yahoo.com.cn

Key Research Laboratory, Wuhan early warning Academy, Wuhan 430019,
} China

\section{Springer}

(c) 2013 Xie et al.; licensee Springer. This is an Open Access article distributed under the terms of the Creative Commons Attribution License (http://creativecommons.org/licenses/by/2.0), which permits unrestricted use, distribution, and reproduction in any medium, provided the original work is properly cited. transmitter and receiver are separated entities, has picked up in recent years.

Monostatic airborne radar with sidelooking array antennas has the desirable property that the relationship between the clutter spatial and Doppler frequencies is both linear and range invariant, i.e., clutter is stationary, thereby enabling its effective suppression using training data from adjacent ranges. However, in many practical applications, for example, monostatic airborne radar with non-sidelooking arrays [4], cylindrical arrays [5], conformal arrays [6], and bistatic airborne radar [7], the clutter spatial-Doppler frequency relationship becomes nonlinear and range dependent, especially at short range, i.e., clutter is non-stationary.

A number of approaches exist to mitigate the impact of clutter non-stationarity on clutter suppression by means of compensation for bistatic airborne radar [8-16]. In [8-12], the mainlobe clutter peak is compensated only from training range cell-to-cell under test (CUT) effectively, and they take little account of compensation for sidelobe clutter. In [13-16], the mainlobe and sidelobe clutter peaks are compensated simultaneously. The above methods are more suitable to bistatic airborne radar with low pulse repetition frequency (PRF), whereas, in the high PRF case (i.e., 
range ambiguity exists), the performance of these methods degrades severely. In this article, a novel clutter suppression method for bistatic airborne radar with range ambiguity is presented, and it completes registration-based compensation based on non-uniform sampling (RBCNS) and the estimated clutter degrees of freedom (DOFs); therefore, the ultimate clutter suppression performance is improved evidently.

This article is organized as follows. In Section 2, the geometry model of bistatic airborne radar is given. In Section 3, the approximate estimation expressions for clutter DOFs in bistatic airborne radar configuration are derived based on the bandwidthlimited signal theory. In Section 4, a novel clutter suppression method is presented for bistatic airborne radar with range ambiguity. The performance of this method is illustrated by computer simulations and conclusions are drawn in Sections 5 and 6, respectively.

\section{Clutter model}

A three-dimensional coordinate system is used throughout this article. Figure 1 shows the coordinate system and bistatic airborne radar parameters, and the main parameters used in this article are listed in Table 1. As shown in Figure 1, the Doppler shift at the receiver of bistatic airborne radar can be expressed by

$$
f_{d}=\frac{1}{\lambda f_{r}}\left[v_{r} \cos \left(\theta_{a r}+\theta_{c r}\right) \cos \varphi_{r}+v_{t} \cos \left(\theta_{X}+\theta_{X t}\right) \cos \varphi_{t}\right],
$$

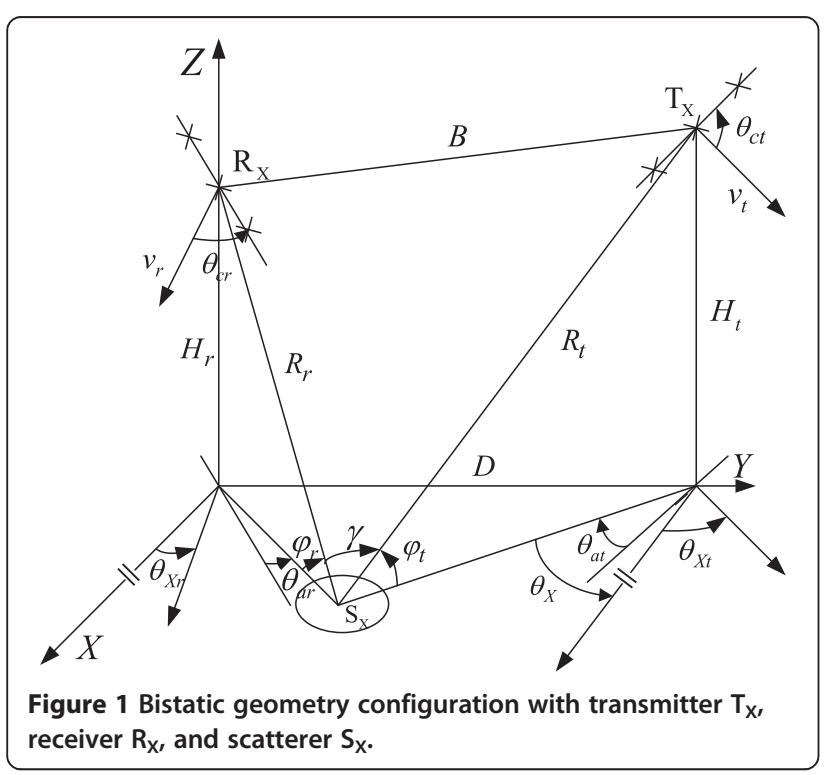

Table 1 The parameter set

\begin{tabular}{ll}
\hline Parameter & Symbol \\
\hline Transmitter, receiver, and scatterer & $T_{X}, R_{X}, S_{X}$ \\
\hline Wavelength & $\lambda$ \\
\hline Pulse repetition interval & $T$ \\
\hline PRF & $f_{r}$ \\
\hline The number of transmitter elements & $M$ \\
\hline The number of receiver elements & $N$ \\
\hline The number of temporal coherent processing pulses & $K$ \\
\hline The length of baseline & $B$ \\
\hline The length of projection of baseline & $D$ \\
\hline The element spacing of transmitter, receiver & $d_{t,} d_{r}$ \\
\hline The heights of transmitter and receiver & $H_{t,} H_{r}$ \\
\hline The slant ranges of the transmitter and receiver & $R_{t,} R_{r}$ \\
\hline The platform velocities of transmitter and receiver & $v_{t,} v_{r}$ \\
\hline The angles between the transmitter and receiver & $\theta_{X t} \theta_{X r}$ \\
velocity and the $X$-axis & $\theta_{c t,} \theta_{c r}$ \\
\hline The crab angles of transmitter and receiver antennas & $\theta_{a t,} \theta_{a r}$ \\
\hline The azimuth angles of transmitter and receiver & $\theta_{X}$ \\
\hline The angles between the line-of-sight of transmitter \\
and the $X$-axis \\
\hline The pitching angles of transmitter and receiver \\
\hline The bistatic angle & $\varphi_{t,} \varphi_{r}$ \\
\hline bistatic range sum & $Y$ \\
\hline
\end{tabular}

where

$$
\sin \varphi_{r}=\frac{H_{r}}{R_{r}}
$$

$\theta_{a r}= \begin{cases}\frac{\pi}{2}-\arccos \left[\frac{D^{2}+R_{B}{ }^{2}-\left(R_{t}^{2}-H_{t}^{2}\right)}{2 D R_{B}}\right]-\theta_{X r}-\theta_{c r}, & \theta_{X} \in(0, \pi] \\ \arccos \left[\frac{D^{2}+R_{B}{ }^{2}-\left(R_{t}^{2}-H_{t}^{2}\right)}{2 D R_{B}}\right]-\theta_{X r}-\theta_{c r}, & \theta_{X} \in(\pi, 2 \pi]\end{cases}$

$$
R_{t}=\sqrt{\left(R_{r}{ }^{2}-H_{r}{ }^{2}\right)+D^{2}-2 \sqrt{\left(R_{r}^{2}-H_{r}^{2}\right)} D \sin \left(\theta_{c r}+\theta_{a r}+\theta_{X r}\right)+H_{t}^{2}}
$$

In this section, the bistatic range is divided into the near and far field. If $R_{r} \leq D$, i.e., the near field, the contour of constant bistatic range sum is a circle of radius $R_{B}$, which center is the projection of receiver onto the $(X, Y)$ plane. Whereas, if $R_{r}>D$, i.e., the far field, the contour of constant bistatic range sum is an ellipse with the projection of transmitter-receiver pair onto the $(X$, $Y$ ) plane at each ellipse foci. Figure 2 shows the above two cases of the contour of constant bistatic range sum. 


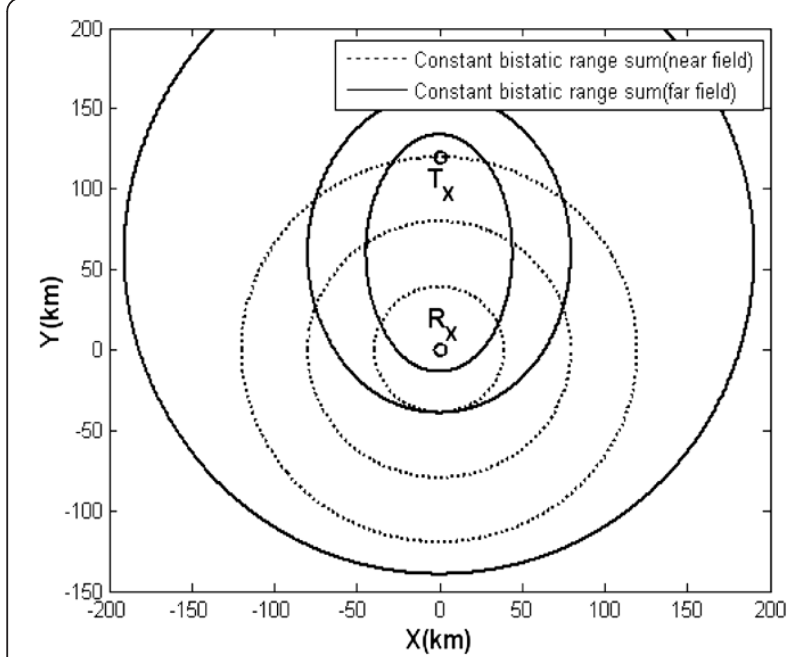

Figure 2 The contour of constant bistatic range sum.

\section{Clutter DOFs estimation}

On the principle of adaptive theory [2], the DOFs of a system should be larger than that of the interference. Therefore, the research on the clutter DOFs is of great importance for STAP. Brennan and Staudaher's rule [17] gives the clutter DOFs for sidelooking ULAs by eigenanalysis of the covariance matrix firstly. For more complex radar configurations, such as overlapping uniform linear subarrays [18], distorted linear and bistatic arrays [19], arbitrary arrays [20], and sparse arrays [21], clutter DOFs are achieved by considering the space-time clutter as an equivalent timelimited sampling of a spatial-frequency band-limited random process. In addition, the clutter local DOFs have been researched in [22].

In [19], the estimation expression of clutter DOFs for bistatic ULA is obtained, however, the sidelooking ULA condition is discussed only when transmitter and receiver move simultaneously. In this section, the estimation expressions of clutter DOFs are extended to the universal case for bistatic array configurations.

As is well known that the echo received during one coherent processing interval (CPI) is equivalent to the spatial bandwidth-limited signal, therefore the temporal extent and bandwidth can be transformed to the spatial effective aperture $L_{\mathrm{ef}}$ and bandwidth $B_{\mathrm{s}}$, and the estimation of clutter DOFs is $\left\lfloor L_{\mathrm{ef}} B_{s}+1\right\rfloor$ based on bandwidth-limited signal theory.

As is shown in Figure 1, the approximate bistatic clutter DOFs corresponding to the bistatic airborne radar configuration is computed by incorporating the effective receiver aperture and the effective transmitter aperture. The effective receiver aperture $L_{r}$ across the CPI is caused by receiver motion only, by contrary, the effective transmitter apertures $L_{t}$ across the CPI are caused by transmitter motion only. So, the spatial effective aperture $L_{\mathrm{ef}}=L_{r}+L_{t}$. The spatial bandwidth is given by

$$
B_{S}=2 \frac{\cos \varphi_{r}}{\lambda}
$$

The approximate bistatic clutter DOFs can be expressed as

$$
\rho=\left\lfloor\left(L_{r}+L_{t}\right) \times B_{S}+1\right\rfloor
$$

\subsection{Receiver moves, and transmitter is stationary}

Using the bistatic configuration and system parameters as shown in Figure 1 and Table 1, respectively, the effective receiver aperture $L_{r}$ is divided into the components of the receiver aperture along and orthogonal to the direction of receiver motion across the CPI, i.e., $L_{r}=L_{r 1}+L_{r 2}$, and they are approximately given by

$$
\begin{aligned}
& L_{r 1}=\left[(N-1) \times\left|\cos \theta_{c r}\right|+(K-1) \beta_{r}\right] d_{r} \\
& L_{r 2}=\left[(N-1) \times\left|\sin \theta_{c r}\right|\right] d_{r}
\end{aligned}
$$

where $\beta_{r}=v_{r} T / d_{r}$. Because transmitter is stationary, the effective transmitter apertures $L_{t}=0$.

In the simulation of this section, the number of clutter eigenvalues is decided based on capturing 99.999\% of the total echo energy. Figure 3 illustrates the true and estimated clutter DOFs versus the number of receiver subarray and the crab angle of receiver antennas for receiver with sidelooking array and non-sidelooking array, respectively. The close agreement between the true and estimated DOFs in Figure 3 can be observed, and the exclusive difference is that the true value is slightly greater than estimated value as shown in Figure 3a, whereas the estimated value is slightly greater as shown in Figure $3 \mathrm{~b}$.

\subsection{Transmitter moves, and receiver is stationary}

Because transmitter moves only, the effective receiver aperture is given by

$$
L_{r}=(N-1) \times d_{r}
$$

The effective transmitter aperture $L_{t}$ is divided into the components of the transmitter aperture along and 

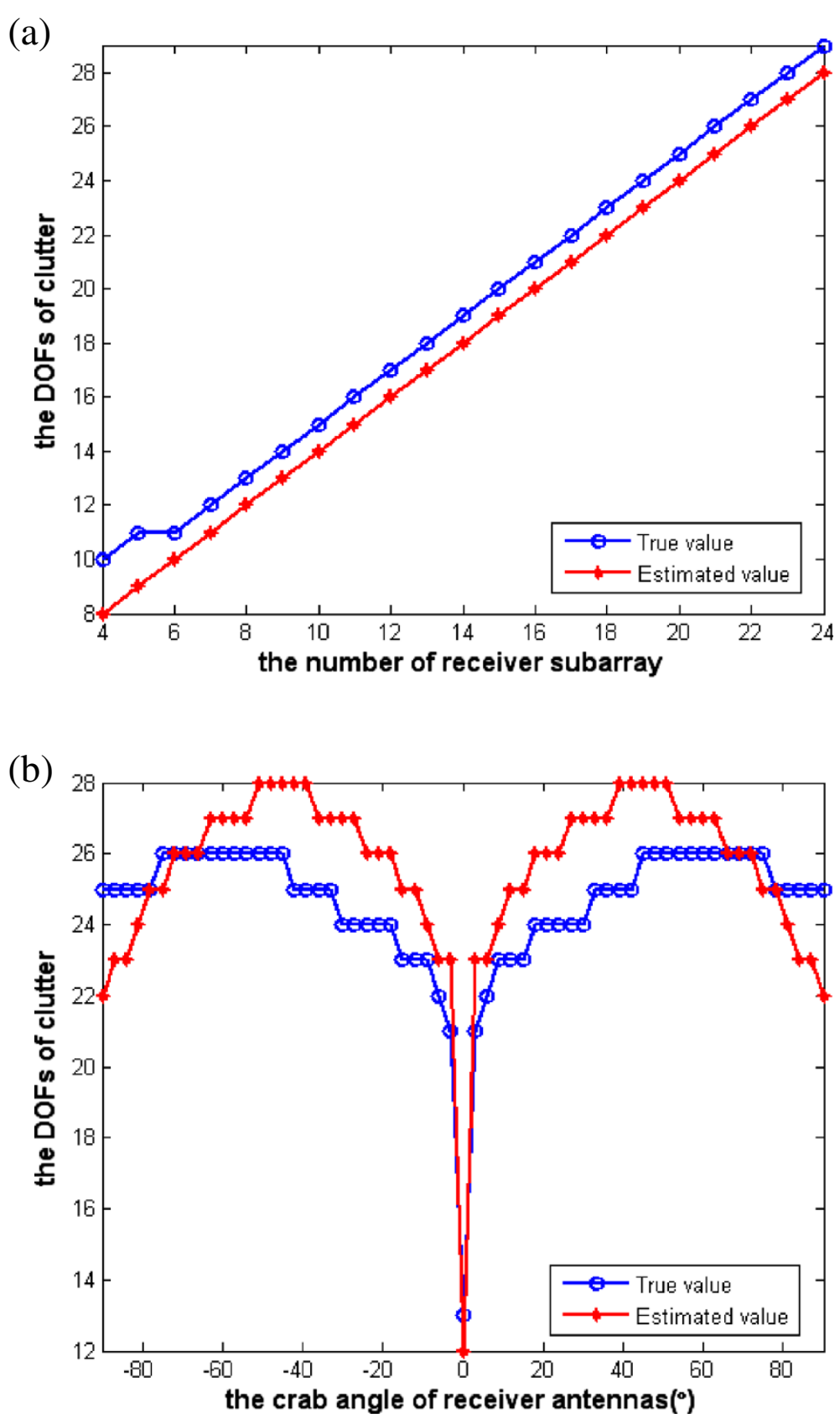

Figure 3 Clutter DOFs comparison of true value (o) and estimated value $\left(^{*}\right)$ in the case of receiver moves, and transmitter is stationary. (a) Receiver with sidelooking array. (b) Receiver with non-sidelooking array.

orthogonal to the direction of receiver motion across the CPI, i.e., $L_{t}=L_{t 1}+L_{t 2}$, and they are approximately given by

$$
\begin{aligned}
& L_{t 1}=(M-1) \beta_{t} d_{t} \times\left|\cos \left(\theta_{X t}-\theta_{X r}-\theta_{c r}\right)\right| \\
& L_{t 2}=(M-1) \beta_{t} d_{t} \times\left|\sin \left(\theta_{X t}-\theta_{X r}-\theta_{c r}\right)\right|
\end{aligned}
$$

where $\beta_{t}=v_{t} T / d_{t}$. Figure 4 illustrates the true and estimated clutter DOFs versus the crab angle of transmitter antennas for receiver with sidelooking array and non-sidelooking array, respectively. The close agreement between the true and estimated DOFs in Figure 4 can be observed, which is analogous to the results of Figure 3. 

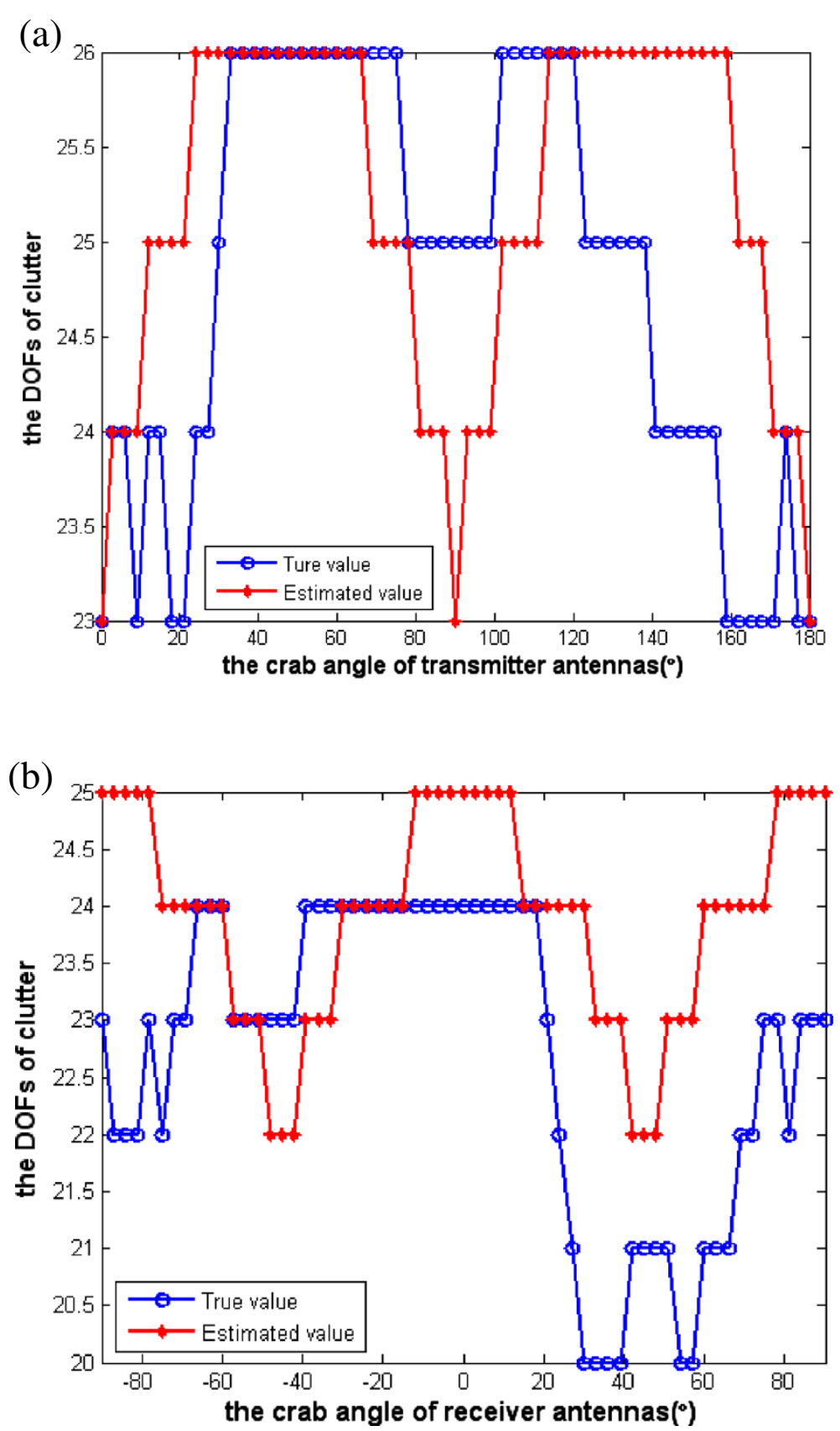

Figure 4 Clutter DOFs comparison of true value (o) and estimated value $\left(^{*}\right)$ in the case of transmitter moves, and receiver is stationary. (a) Receiver with sidelooking array. (b) Receiver with non-sidelooking array.

3.3. Transmitter and receiver move simultaneously 3.3.1. Receiver with sidelooking arrays

Analogously to Section 3.2, the effective receiver aperture is given by

$$
L_{r}=\left[(N-1)+(M-1) \beta_{r}\right] d_{r}
$$

The effective transmitter aperture $L_{t 1}$ and $L_{t 2}$ are given by

$$
\begin{aligned}
& L_{t 1}=(M-1) \beta_{t} d_{t} \times \cos \left(\theta_{X t}-\theta_{X r}\right) \\
& L_{t 2}=(M-1) \beta_{t} d_{t} \times \sin \left(\theta_{X t}-\theta_{X r}\right)
\end{aligned}
$$



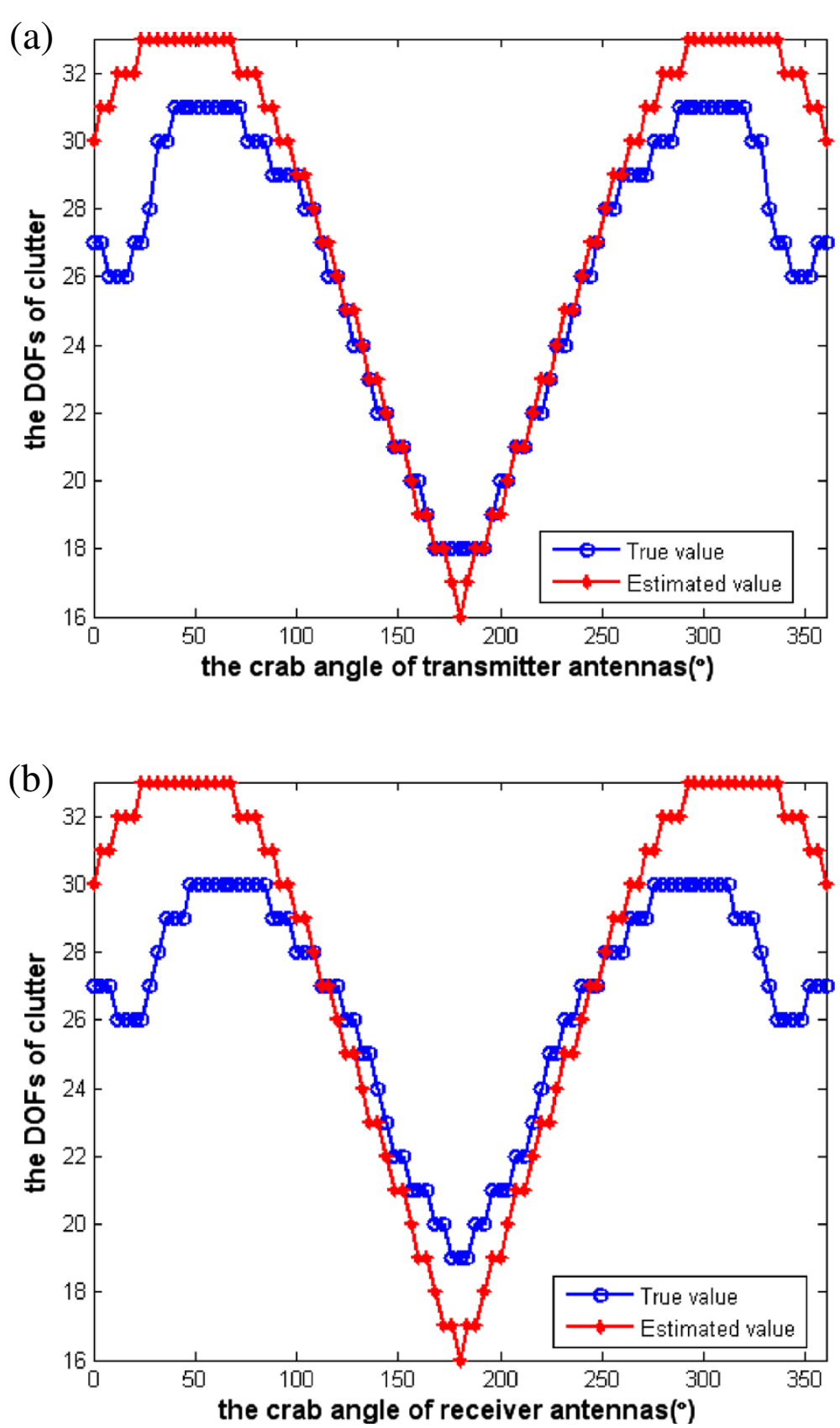

Figure 5 Clutter DOFs comparison of true value (o) and estimated value $\left(^{*}\right)$ in the case of receiver with sidelooking array. (a) The velocity direction of transmitter changes. (b) The velocity direction of receiver changes.

Figure 5 illustrates the true and estimated clutter DOFs versus the crab angle of transmitter antennas and receiver antennas, respectively. The analogous results can be obtained that the estimated clutter DOFs are close to the true value.

\subsubsection{Receiver with non-sidelooking arrays}

When receiver is with non-sidelooking arrays, the effective receiver aperture is the same as in Equation
(12). The effective transmitter aperture $L_{t 1}$ and $L_{t 2}$ are given by

$$
\begin{aligned}
& L_{t 1}=(M-1) \beta_{t} d_{t} \times\left|\cos \left(\theta_{X t}-\theta_{X r}-\theta_{c r}\right)\right| \\
& L_{t 2}=(M-1) \beta_{t} d_{t} \times\left|\sin \left(\theta_{X t}-\theta_{X r}-\theta_{c r}\right)\right|
\end{aligned}
$$

Figure 6 illustrates the true and estimated clutter DOFs versus the crab angle of receiver antennas for $\theta_{X r}=$ 

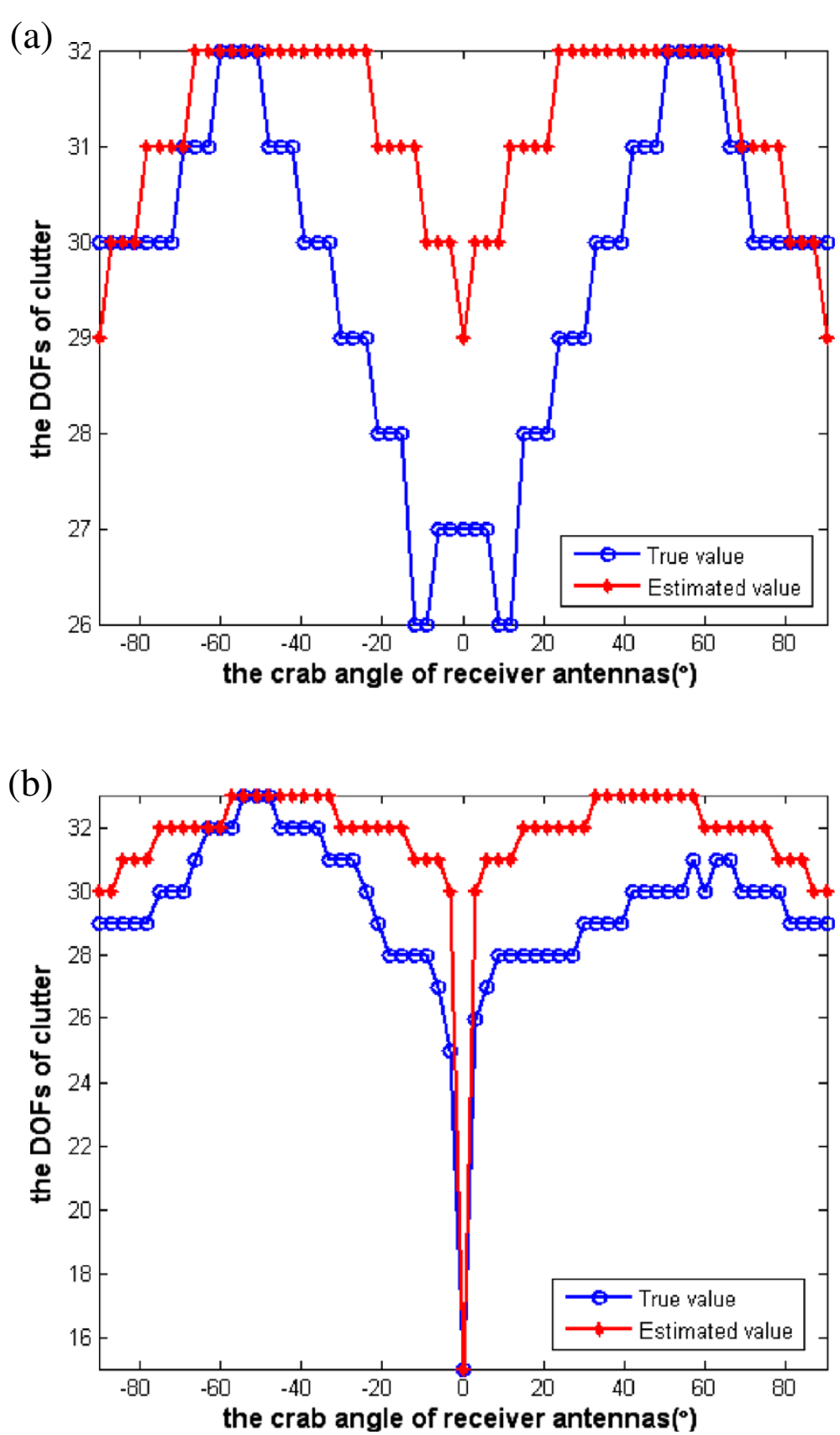

Figure 6 Clutter DOFs comparison of true value (o) and estimated value $\left(^{*}\right)$ in the case of receiver with non-sidelooking array. (a) $\theta_{x_{r}}=0^{\circ}, \theta_{x_{t}}=0^{\circ}$. (b) $\theta_{x_{r}}=90^{\circ}, \theta_{x_{t}}=90^{\circ}$.

$0^{\circ}, \theta_{X t}=0^{\circ}$, and $\theta_{X r}=90^{\circ}, \theta_{X t}=90^{\circ}$, respectively. The results are the same as that of receiver with sidelooking arrays.

In this section, extensive simulations have been performed for several different bistatic configurations, and they indicate that the bistatic clutter DOFs can be estimated to within $6 \%$ of their true value using the presented formulas.

\section{Range ambiguity clutter suppression}

A crucial aspect of the effectiveness of any STAP system is the training and updating of the clutter covariance matrix. Most of the reported advantages of an STAP system accrue from an appropriate knowledge of the clutter covariance matrix. Inherent in its estimation is the assumption about the stationarity of clutter. However, for almost bistatic airborne configurations, the 


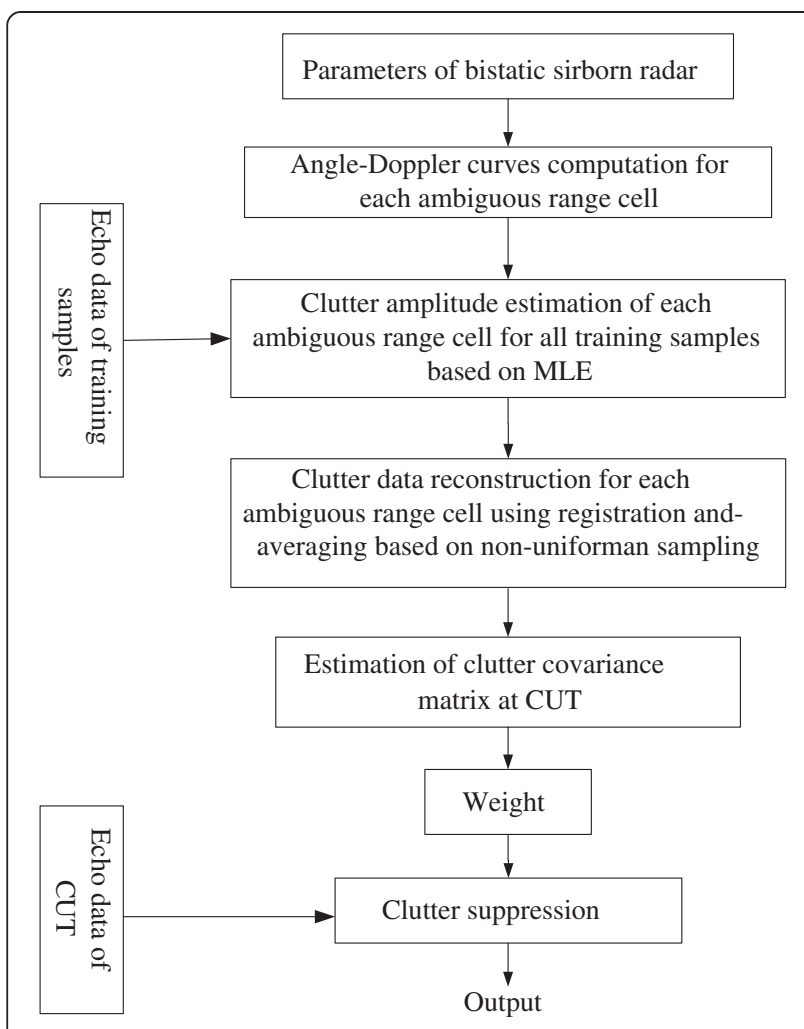

Figure 7 Block diagram of processing steps for RBCNS method.

clutter space-time spectrum changes between range gates, i.e., non-stationarity, leading to significant degradations in performance for STAP, especially for range ambiguity case.

In this section, we present a novel method, i.e., RBCNS, to achieve clutter suppression for bistatic airborne radar with range ambiguity. The RBCNS method consists of four steps: (1) clutter ridges of various ambiguous ranges at all training cell are determined according to the corresponding angle-Doppler curves using the system parameters of bistatic airborne radar. (2) Clutter amplitude of each ambiguous range is estimated for all training samples based on maximum likelihood estimation (MLE) and the estimated clutter DOFs. (3) Clutter data of each ambiguous range at CUT is reconstructed using registration-and-averaging based on non-uniform sampling. (4) The clutter covariance matrix at CUT is estimated using the reconstructed clutter data, and clutter suppression is achieved. Figure 7 shows the block diagram of the RBCNS method. A detailed description of the RBCNS method is as follows.

Let us consider the signal received by a bistatic airborne radar, and the relative movement between the transmitter and the receiver across the CPI is ignored. The bistatic system geometry parameters are assumed to be provided by INS accurately. Each CPI consists of $K$ pulses. For each pulse, one data sample at the range of interest is taken at each of the $N$ receiving elements. The $N K \times 1$ sample for the received clutter echo corresponding to the range of interest under range ambiguity situation is denoted by $X$, which is given by

$$
\boldsymbol{X}=\sum_{l=1}^{L} \sum_{i=1}^{N_{c}} a_{l i} \boldsymbol{S}_{l i}
$$

where $L$ is the range ambiguity degree, $N_{c}$ is the number of clutter patches contributing to the signal, $a_{l i}$ and $S_{l i}$ are the complex amplitude of the signal and spatial-temporal steering vector corresponding to the $i$ th clutter patch for the $l$ th ambiguous range cell. Equation (17) can be rewritten in matrix notation as

$$
\boldsymbol{X}=\sum_{l=1}^{L} \boldsymbol{S}_{l} \boldsymbol{a}_{l}
$$

where $\boldsymbol{S}_{l}=\left[\begin{array}{llll}\boldsymbol{S}_{l 1} & \boldsymbol{S}_{l 2} & \cdots & \boldsymbol{S}_{l N_{c}}\end{array}\right], \boldsymbol{a}_{l}=\left[\begin{array}{llll}a_{l 1} & a_{l 2} & \cdots & a_{l N_{c}}\end{array}\right]^{\mathrm{T}}$ , T represents the transpose of a matrix. In Equation (18), the number of clutter cell $N_{c}$ is so large that the columns of $S_{l}$ are correlated. Assume the column rank of $S_{l}$ be $J$, i.e., the number of clutter DOFs, and they can be estimated according to the estimation formulas in Section 3. For the $l$ th ambiguous range cell, the $J_{l}$ points with maximum amplitude should be selected to denote the information of clutter. So $X$ can also be expressed by

$$
\boldsymbol{X}=\sum_{l=1}^{L} \widetilde{\boldsymbol{S}}_{l} \widetilde{\boldsymbol{a}}_{l}
$$

where $\widetilde{\boldsymbol{S}}_{l}=\left[\begin{array}{llll}\boldsymbol{S}_{l n_{1}} & \boldsymbol{S}_{l n_{2}} & \cdots & \boldsymbol{S}_{l n_{l}}\end{array}\right], \widetilde{\boldsymbol{a}}_{l}=\left[\begin{array}{llll}a_{l n_{1}} & a_{l n_{2}} & \cdots & a_{l n_{l}}\end{array}\right]^{\mathrm{T}}$. Equation (19) indicates that the clutter snapshot $X$ consists of $J_{l}$ uncorrelated space-time steering vectors. Given the assumptions above, the unknown parameters in Equation (19) are $\widetilde{\mathbf{a}}_{l}$, i.e., the scattering coefficient of clutter patch.

Conventional parameter estimation methods include periodogram, adaptive matched filter, least squares estimation, etc. In this section, the MLE is applied. The estimated complex amplitude of clutter corresponding to the $l$ th ambiguous range cell based on the MLE is

$$
\hat{\widetilde{\boldsymbol{a}}}_{l}=\left(\widetilde{\boldsymbol{S}}_{l}^{\mathrm{H}} \boldsymbol{R}_{n}^{-1} \widetilde{\boldsymbol{S}}_{l}+\boldsymbol{R}_{\widetilde{a}_{l}}^{-1}\right)^{-1} \widetilde{\boldsymbol{S}}_{l}^{\mathrm{H}} \boldsymbol{R}_{n}^{-1} \boldsymbol{X}
$$

where $\boldsymbol{R}_{\widetilde{a}_{l}}$ takes proportional to the identity matrix $\operatorname{diag}\left\{\begin{array}{llll}a_{l n_{1}}^{2} & a_{l_{n_{2}}}^{2} & \cdots & a_{l n_{n_{l}}}^{2}\end{array}\right\}, \quad \boldsymbol{R}_{n}$ is the covariance 
matrix of the thermal noise, and $\mathrm{H}$ represents the conjugate transpose of a matrix. It is important that only $J_{l}$ amplitude of uncorrelated clutter patches is estimated, and it causes non-uniform sampling and low computational load. The locations of clutter ridge corresponding to each ambiguous range cell for every training data can be derived based on the system parameters of bistatic airborne radar.

Using the registration-and-average technique [15], the clutter snapshot of the $l$ th ambiguous range at the CUT according to Equation (19) is

$$
\hat{\boldsymbol{X}}_{l 0} \approx \tilde{\boldsymbol{S}}_{l 0} \hat{\tilde{\boldsymbol{a}}}_{l 0}
$$

where $\widetilde{\boldsymbol{S}}_{l 0}$ is the space-time steering vector at the CUT, and $\hat{\tilde{\boldsymbol{a}}}_{l 0}$ is the averaged clutter amplitude corresponding to $\widetilde{\boldsymbol{S}}_{l 0}$. So, the clutter covariance matrix $\hat{\boldsymbol{R}}$ estimated at the CUT is

$$
\hat{\boldsymbol{R}}=\sum_{l=1}^{L} \hat{\boldsymbol{X}}_{l 0} \hat{\boldsymbol{X}}_{l 0}^{H}
$$

and the space-time adaptive weight is

$$
\boldsymbol{W}=\mu\left(\hat{\boldsymbol{R}}+\boldsymbol{R}_{n}\right)^{-1} \boldsymbol{S}
$$

where $S$ is the space-time steering vector, and $\mu$ is the constant.

In the RBCNS method, the following two points are stressed. (1) The prior knowledge of system parameters of bistatic airborne radar is needed in order to achieve the estimation of complex amplitude of ambiguous clutter, therefore, the mismatch between actual system parameters and those given by Inertial Navigation System (INS) in practice will result in performance degradation slightly. (2)The advantage of this presented method is the cancellation of ambiguous clutter, and it is completed using the respective estimation of ambiguous clutter snapshot in Equation (21).

\section{Simulations}

A simulation is performed with a bistatic array, where the transmitter and receiver are traveling in the same direction in parallel. The primary parameters in the simulation are assumed to be as follows. The number of receiver subarray $N=8$, temporal dimension of clutter filter $K=16$, transmitter and receiver platform velocities $v_{t}=v_{r}=140 \mathrm{~m} / \mathrm{s}$, radar wavelength $\lambda=$ $0.23 \mathrm{~m}$, system bandwidth $B=1 \mathrm{MHz}$, the input clutter-to-noise ratio $(\mathrm{CNR})=60 \mathrm{~dB}$, two bistatic

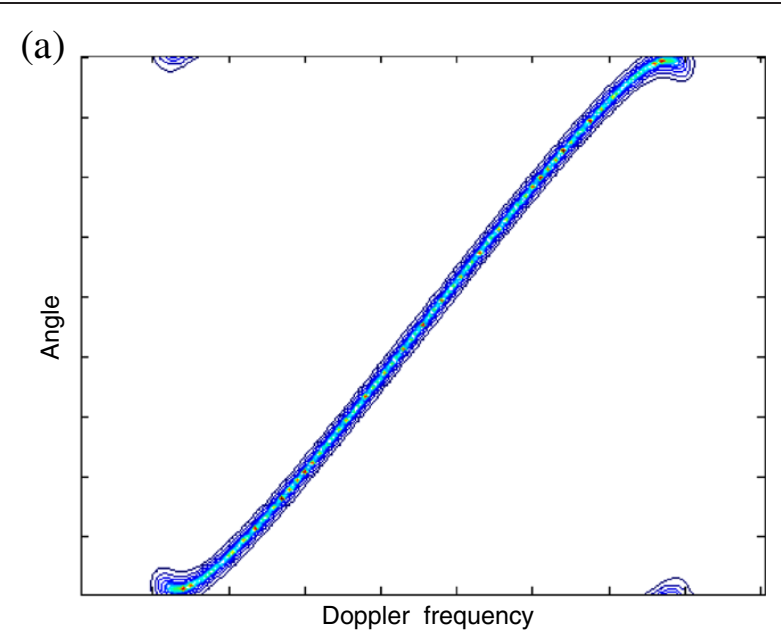

(b)

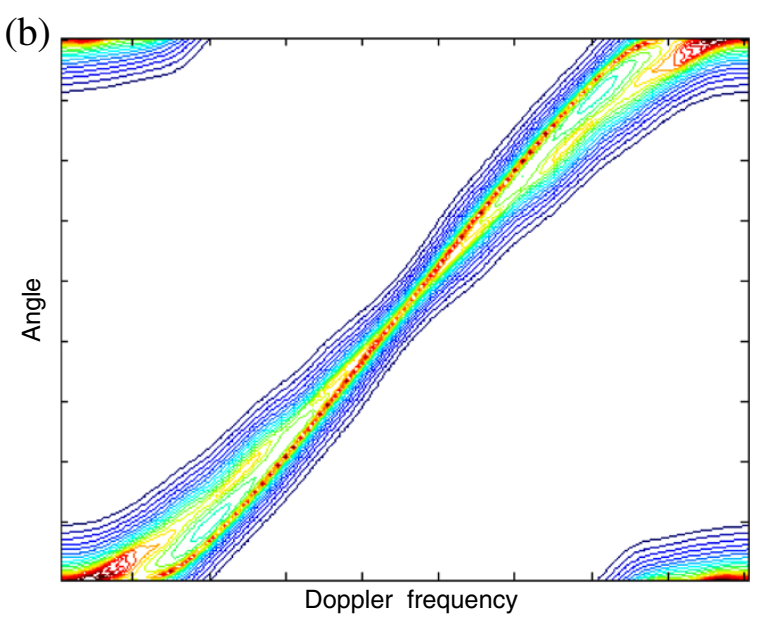

Figure 8 Clutter power spectrum distribution at CUT. (a) Clutter power spectrum without range ambiguity. (b) Clutter power spectrum with range ambiguity.

maximum range sum situations are assumed including $400 \mathrm{~km}$ (i.e., the degree of range ambiguity is 3) and $700 \mathrm{~km}$ (i.e., the degree of range ambiguity is 5). The averaged simulation results are as follows via Monte Carlo experiment with 100 times.

Figure 8 shows clutter power spectrum distribution at CUT with and without range ambiguity, where the effect of backlobe is not considered which can be suppressed by means of a metal reflector in most practical radar applications. The range ambiguity causes the clutter spectrum broadened severely as shown in Figure $8 \mathrm{~b}$ compared with the case without range ambiguity in Figure $8 \mathrm{a}$. The reason is that additional range ambiguous clutter returns, i.e., multiple-time-around clutter, are received simultaneously. 


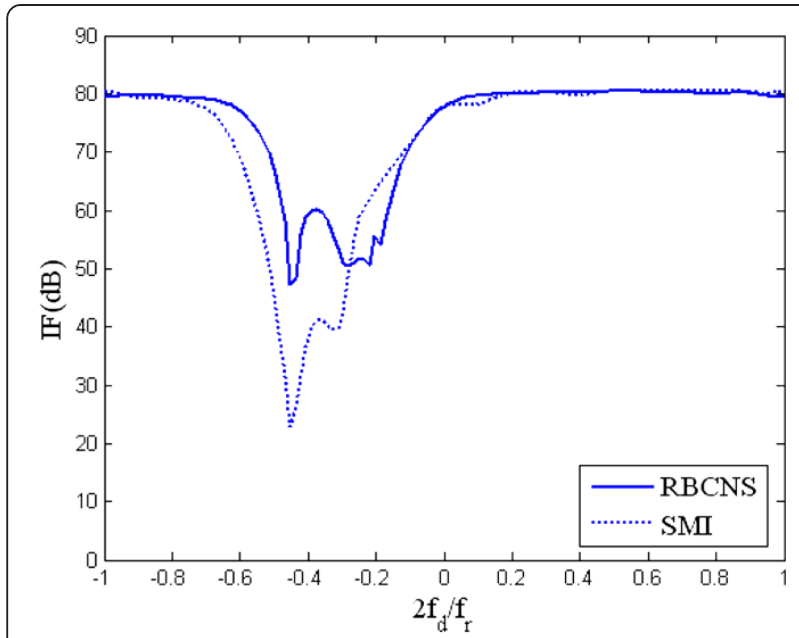

Figure 9 IF versus normalized Doppler for three degrees of range ambiguity.

The clutter suppression performance of the proposed RBCNS method degrades about $3 \mathrm{~dB}$ at the sidelobe region, and $5 \mathrm{~dB}$ at the mainlobe region compared with the clairvoyant filter (i.e., known Cov.) by a number of simulation results. The results are not given in the following experiments due to the limit of length.

\subsection{Experiment 1 (the comparison of improvement factors)}

Figures 9 and 10 show improvement factor (IF) values against normalized Doppler frequency for a target located at the look direction, and clutter

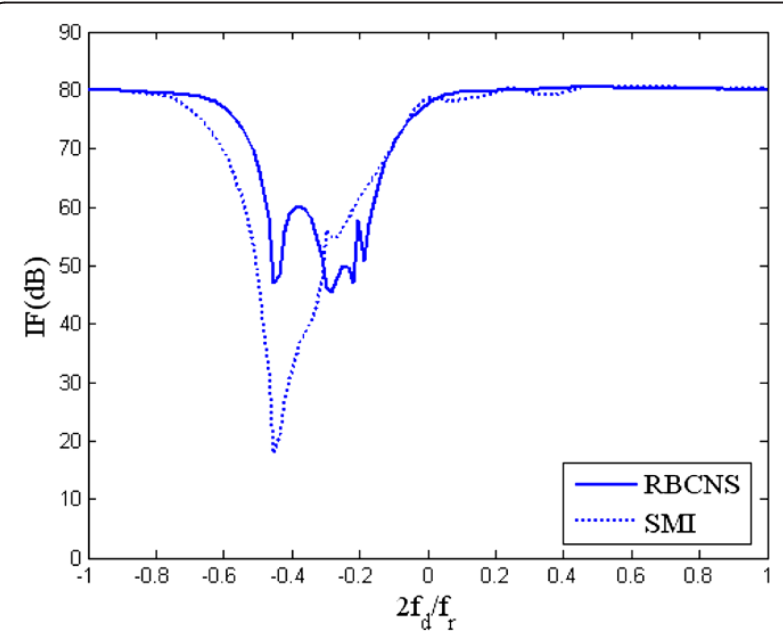

Figure 10 IF versus normalized Doppler for five degrees of range ambiguity. suppression performance of conventional SMI [1] and RBCNS are compared, where the SMI method uses the estimated covariance matrix without any preprocessing.

From Figures 9 and 10, it can be noticed that the clutter notch is severely broadened for conventional SMI method, and the performance of RBCNS is found better than that of SMI evidently in mainlobe clutter region except some especial narrow clutter band. The reason is that range ambiguous clutter echoes have a different Doppler frequency relative to the primary clutter for bistatic configuration, which causes additional clutter notches in the Doppler response of the space-time filter.

The primary clutter and range ambiguous clutter are handled as a whole for conventional SMI method, whereas they are eliminated separately for RBCNS method. It leads to that one broad clutter notch is instead of about two narrow clutter notches, where the first notch is the one due to primary clutter at near range, and the second notch is due to multiple range ambiguous clutter returns which come from farther distances. Since the clutter Doppler frequency changes more quickly at near range for bistatic configuration, and most of the multiple range ambiguous arrivals come from large ranges, therefore they altogether have about the same Doppler frequency so that their influence sums up in one additional clutter notch as shown in Figures 9 and 10 .

\subsection{Experiment 2 (the comparison of output signal-to-clutter-plus-noise ratio)}

To illustrate the detection performance of clutter suppression methods including SMI and RBCNS, we inject one moving target. The artificial target is injected in range cell 51, i.e., the bistatic range is $130 \mathrm{~km}$. Other parameters of the injected target are given as follows. Signal-to-noise ratio $(\mathrm{SNR})=10 \mathrm{~dB}$, normalized Doppler frequency $2 f_{d} / f_{r}=-0.5$.

The output signal-to-clutter-plus-noise ratio (SCNR) at the CUT is

$$
\mathrm{SCNR}=\frac{\left|\boldsymbol{W}^{\mathrm{H}} \boldsymbol{S}\right|^{2}}{\boldsymbol{W}^{\mathrm{H}} \boldsymbol{R} \boldsymbol{W}}
$$

where $W$ denotes adaptive weight of different clutter suppression method, $\boldsymbol{R}$ and $S$ denote clutter covariance matrix and space-time steering vector, respectively, all for the CUT.

Figures 11 and 12 plot the detection results of different methods, and it is of the output clutter residual as a 

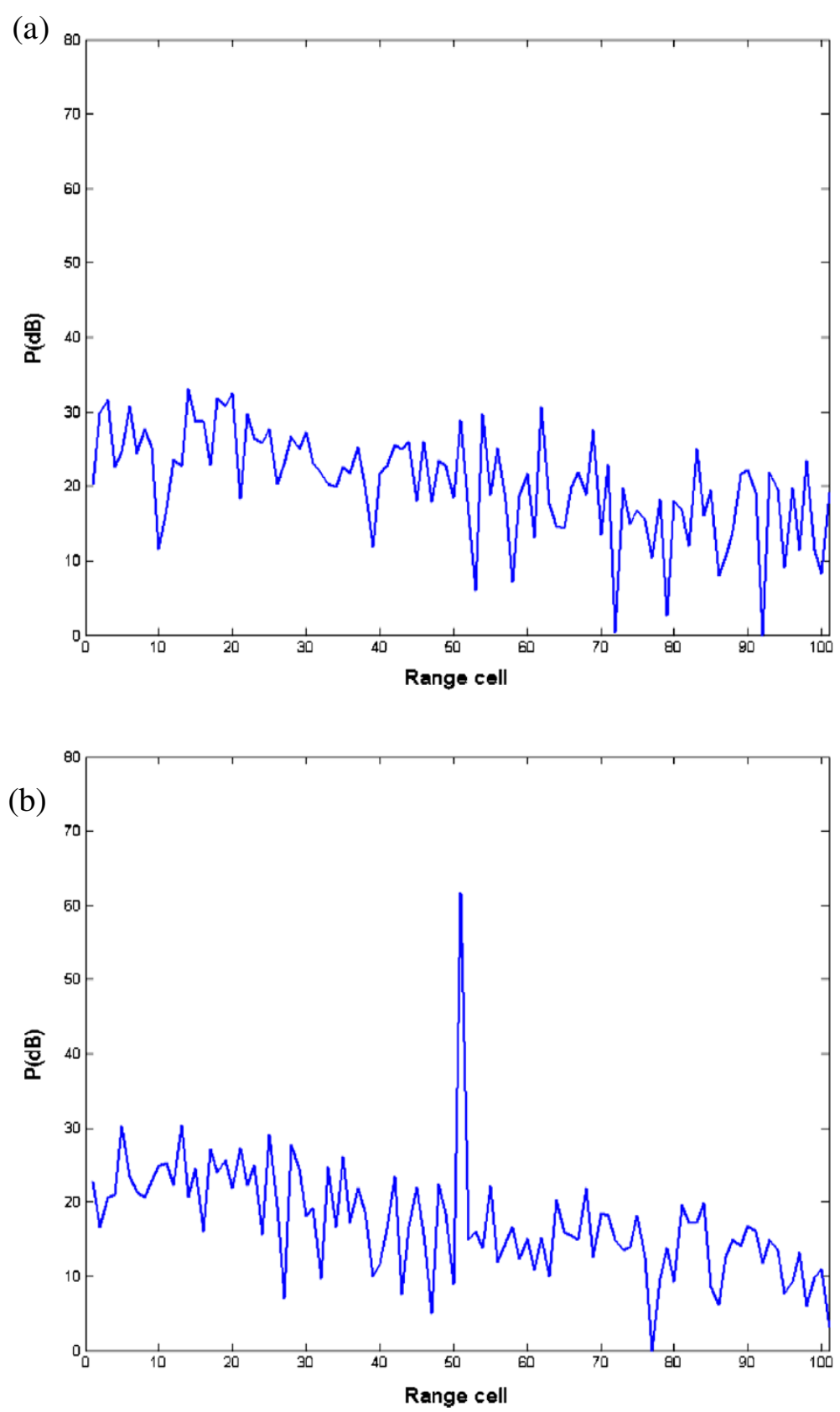

Figure 11 Output clutter residual comparison of two different methods for three degrees of range ambiguity. (a) SMI. (b) RBCNS.

function of range. From Figures 11 and 12, the output SCNR improvement of the RBCNS method relative to SMI is quite evident, and the results are consistent with those in Figures 9 and 10.

5.3. Experiment 3 (the comparison of Doppler blind zone) An issue of importance in STAP is the Doppler blind zone. The associated Doppler blind zones of two methods are given in Table 2, wherein the Doppler blind zone of RBCNS is obviously less than that of SMI under two different degrees of range ambiguity situations. The decreased Doppler blind zone is derived from the mainlobe region mainly, and it causes less minimum detected velocity and improved ground moving target indication performance for the RBCNS method. 
(a)

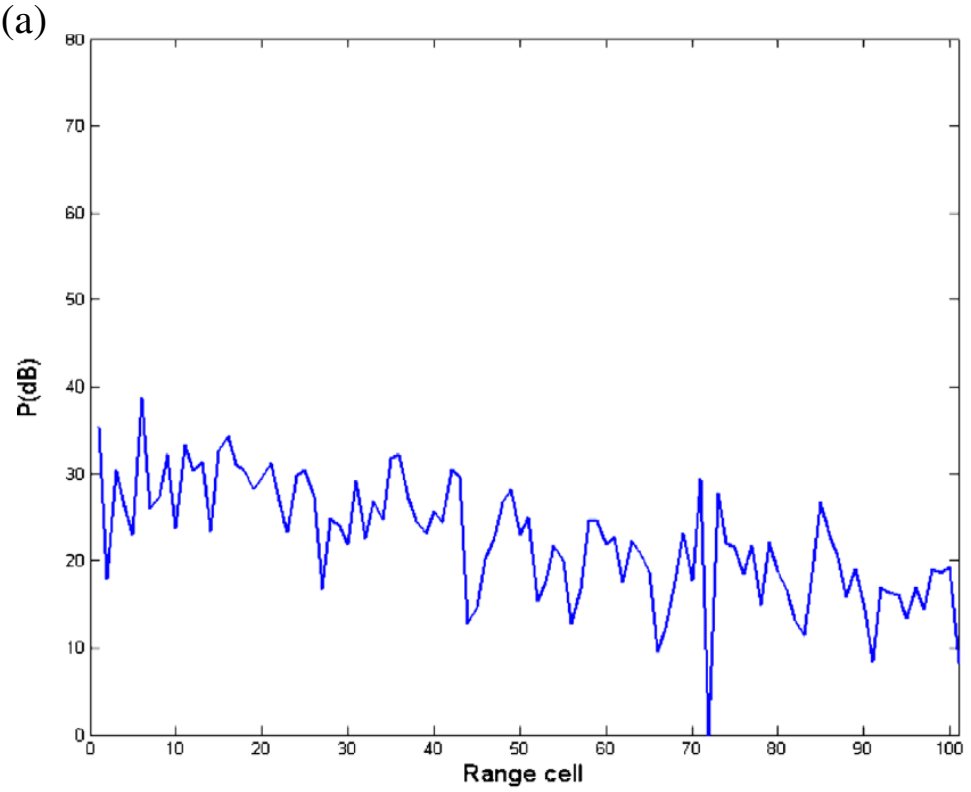

(b)

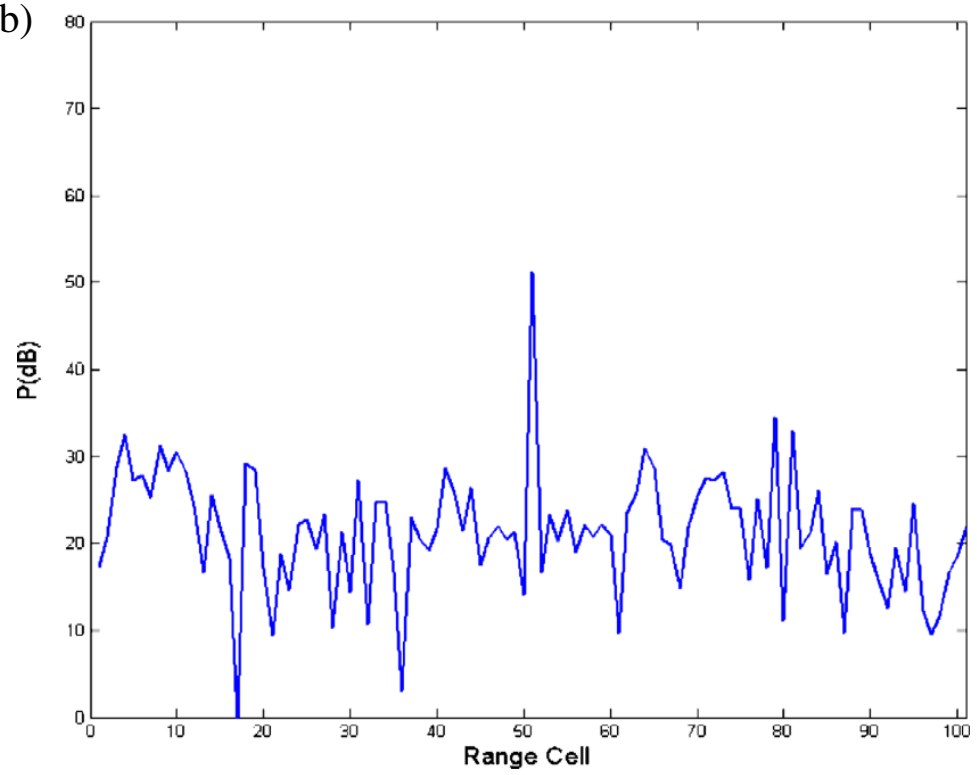

Figure 12 Output clutter residual comparison of two different methods for five degrees of range ambiguity. (a) SMI. (b) RBCNS.

5.4. Experiment 4 (the comparison of computation load) In this section, the computation load of the proposed method is analyzed in a form of add and multiplication operations based on the general parameters of radar system. The comparison is presented in Table 3, where the clutter DOFs $J$ is assumed to be 32 for

Table 2 Doppler blind zone comparison of two methods

\begin{tabular}{lll}
\hline The degree of range ambiguity & SMI (\%) & RBCNS (\%) \\
\hline 3 & 18 & 4.25 \\
\hline 5 & 20 & 4.75 \\
\hline
\end{tabular}

various ambiguous range for convenience, and the training sample value $P=2 N K$ for the SMI method. As is shown in Table 3, the proposed method has a heavy computation burden about one quantity level than SMI-based method due to the non-stationarity compensation for range ambiguity clutter.

Table 3 The comparison of computation load

\begin{tabular}{lll}
\hline Method & Computation load & $\begin{array}{l}\text { The general parameters } \\
\text { of radar system case }\end{array}$ \\
\hline RBCNS & $L\left(2(N K)^{3}+3 J(N K)^{2}+2 J^{3}+J^{2} N K\right)$ & $1.79 \times 10^{7}$ \\
\hline SMI & $(N K)^{3}+P(N K)^{2}$ & $6.29 \times 10^{6}$ \\
\hline
\end{tabular}




\section{Conclusions}

The non-stationarity of the response of a bistatic airborne radar to clutter as a function of range makes it difficult to obtain reliable estimates of the covariance matrix of the array output. The quality of this covariance estimation has a strong impact on the performance of the STAP algorithm. Using the conventional sample covariance matrix significantly degrades performance at short range. In this article, the clutter suppression techniques for further improving bistatic airborne radar STAP performance with range ambiguity are examined, and the presented method is quite effective in eliminating or reducing the performance loss due to the nonstationarity of range ambiguity clutter. This comes at the cost of increased computational requirements.

In this article, we give emphasis to the effect of nonstationarity due to specific bistatic array configuration. In practice, except for clutter non-stationarity, the nonhomogeneity of clutter [23] caused by environments is inevitable, such as power non-homogeneity, interference target, discrete interference, etc., and it will cause that the variation of clutter statistics at range becomes more serious, and clutter DOFs are further increased. Both nonstationarity and non-homogeneity of clutter suppression will be the field of future research.

\section{Competing interests}

The authors declare that they have no competing interests.

\section{Acknowledgments}

The authors would like to thank the anonymous reviewers for providing many detailed and helpful suggestions which lead to a better presentation of this paper. This study was presented in part at the National Nature Science Foundation of China under Contract nos. 61102169 and 60925005.

Received: 12 June 2012 Accepted: 4 March 2013

Published: 10 April 2013

\section{References}

1. J Ward, Space-time adaptive processing for airborne radar, Technical report (MIT Lincoln Laboratory, Lexington, MA, 1994)

2. R Klemm, Principles of Space-Time Adaptive Processing, 2nd edn., IEE Radar, Sonar, Navigation, and Avionics 9 (IEE Publishing, London, UK, 2002)

3. J Guerci, Space-Time Adaptive Processing for Radars (Artech House, London, UK, 2003)

4. YL Wang, YN Peng, Z Bao, Space-time adaptive processing for airborne radar with various array orientations. IEE Proc. Radar Sonar Navigat. 144(6), 330-340 (1997)

5. WC Xie, YL Wang, STAP for airborne radar with cylindrical phased array antennas. Signal Process. 89(6), 883-893 (2009)

6. WC Xie, KQ Duan, F Gao, YL Wang, ZH Zhang, Clutter suppression for airborne phased radar with conformal arrays by least squares estimation. Signal Process. 91(7), 1665-1669 (2011)

7. R Klemm, Comparison between monostatic and bistatic configurations for STAP. IEEE Trans. Aerosp. Electron. Syst. 36(2), 596-608 (2000)

8. GK Borsari, Mitigating effects on STAP processing caused by an inclined array, in Proceedings of the IEEE National Radar Conference (IEEE Press, USA, 1998), pp. 1-7

9. O Kreyenkamp, R Klemm, Doppler compensation in forwarding STAP radar. IEE Proc. Radar Sonar Navigat. 148(5), 253-258 (2001)

10. B Himed, YH Zhang, A Hajjar, STAP with angle-doppler compensation for bistatic airborne radar, in Proceedings of the IEEE National Radar Conference (IEEE Press, USA, 2002), pp. 311-317
11. W Melvin, B Himed, M Davis, Doubly adaptive bistatic clutter filtering, in Proceedings of the IEEE National Radar Conference (IEEE Press, USA, 2003), pp. $171-178$

12. C Lim, E Aboutanios, B Mulgrew, Modified JDL with Doppler compensation for airborne bistatic radar, in Proceedings of the IEEE International Radar Conference (IEEE Press, USA, 2005), pp. 854-858

13. C Lim, B Mulgrew, Filter banks based JDL with angle and separate Doppler compensation for airborne bistatic radar, in Proceedings of the International Radar Symposium (IEEE Press, USA, 2005), pp. 1-4

14. F Pearson, G Borsari, Simulation and analysis of adaptive interference suppression for bistatic surveillance radars, in Proceedings of the Adaptive Sensor Array Processing Workshop (MIT Lincoln Laboratory, USA, 2001), pp. 1-22

15. F Lapierre, M Droogenbroeck, J Verly, New solutions to the problem of range dependence in bistatic STAP radars, in Proceedings of the IEEE National Radar Conference (IEEE Press, USA, 2003), pp. 452-459

16. A Jaffer, B Himed, P Ho, Estimation of range-dependent clutter covariance by configuration system parameter estimation, in Proceedings of the IEEE National Radar Conference (IEEE Press, USA, 2005), pp. 596-601

17. LE Brennan, FM Staudaher, Subclutter visibility demonstration, Technical report, Adaptive Sensors Incorporated, 1992. March

18. Q Zhang, WB Mikhael, Estimation of the clutter rank in the case of subarraying for space-time adaptive processing. Electron. Lett. 33(5), 419-420 (1997)

19. V Varadarajan, JL Krolik, Joint space-time interpolation for distorted linear and bistatic array geometries. IEEE Trans. Signal Process. 56(3), 848-860 (2006)

20. NA Goodman, JM Stiles, On clutter rank observed by arbitrary arrays. IEEE Trans. Signal Process. 55(1), 178-186 (2007)

21. Y Wu, J Tang, YN Peng, Clutter rank of multi-dimensional sparse array radar, in Proceedings of the IEEE Radar Conference (IEEE Press, Boston, USA, 2007), pp. 463-468

22. ZH Zhang, WC Xie, WD Hu, WX Yu, Local degrees of freedom of airborne array radar clutter for STAP. IEEE Geosci. Remote Sens. Lett. 6(1), 97-101 (2009)

23. W Melvin, Space-time adaptive radar performance in heterogeneous clutter. IEEE Trans. Aerosp. Electron. Syst. 36(2), 621-633 (2000)

doi:10.1186/1687-6180-2013-75

Cite this article as: Xie et al:: Range ambiguity clutter suppression for bistatic STAP radar. EURASIP Journal on Advances in Signal Processing 2013 2013:75.

\section{Submit your manuscript to a SpringerOpen ${ }^{\circ}$ journal and benefit from:}

- Convenient online submission

- Rigorous peer review

- Immediate publication on acceptance

- Open access: articles freely available online

- High visibility within the field

- Retaining the copyright to your article

Submit your next manuscript at $>$ springeropen.com 